\section{PRE-SEASON HIP/GROIN STRENGTH AND RATINGS OF HEALTH ARE ASSOCIATED WITH PROSPECTIVE INJURY IN PROFESSIONAL FOOTBALLERS}

${ }^{1}$ Matthew Bourne*, ${ }^{2}$ Morgan Williams, ${ }^{3}$ Ryan Timmins, ${ }^{4}$ Tania Pizzari. ${ }^{1}$ School of Allied Health Sciences, Griffith University, Australia; ${ }^{2}$ School of Health, Sport and Professional Practice, University of South Wales, Wales; ${ }^{3}$ School of Behavioural and Health Sciences, Australian Catholic University, Australia; ${ }^{4}$ La Trobe Sport \& Exercise Medicine Research Centre, La Trobe University, Australia

\subsection{6/bjsports-2019-scandinavianabs.4}

Background Hip and groin injuries are a significant cause of time lost from training and competition in elite football.

Purpose To explore the association between hip/groin injury in professional footballers and pre-season assessment of 1) hip adductor and abductor strength, and 2) the Copenhagen Hip and Groin Outcome Score (HAGOS).

Study Design Prospective cohort.

Methods In total, $\mathrm{n}=204$ elite male footballers from ten professional clubs underwent assessments of hip adductor and abductor strength and completed the HAGOS in pre-season. In-season hip/groin injuries were assessed and reported by team medical staff. Data reduction was conducted using principal component analysis. The subsequent factor component for HAGOS, and three factor components for strength and imbalance measures, were entered with age into a multivariate logistic regression model to determine their association with prospectively occurring hip/groin injury.

Results Twenty-four players suffered at least one hip/groin injury throughout the 2017-18 competitive season. The principal component for between-limb abduction imbalance (peak strength in the preferred [kicking] limb - nonpreferred limb) $(\mathrm{OR}=0.61,95 \% \mathrm{CI}=0.40$ to 0.92$)$, and the principal component for HAGOS $(\mathrm{OR}=0.80,95 \% \mathrm{CI}=0.64$ to 0.99 ) were independently associated with a reduced risk of future hip/groin injury. Receiver operator curve analysis of the whole model revealed an area under the curve of 0.76 .

Conclusion These data demonstrate that a lower likelihood of prospective hip/groin injury during the season was associated with a hip abduction imbalance that favoured the preferred kicking limb and higher HAGOS obtained at pre-season.

\section{FRONTAL PLANE FEMORAL ADDUCTION DURING SINGLE-LEG LANDING AND LOW BACK PAIN IN YOUNG ATHLETES: A PROSPECTIVE PROFITS COHORT STUDY}

\footnotetext{
1,2 Marleena Rossi ${ }^{*}, 1,3,4,5$ Kati Pasanen, ${ }^{2}$ Ari Heinonen, ${ }^{6}$ Sami Äyrämö, ${ }^{3,4}$ Anu Räisänen, ${ }^{1}$ Mari Leppänen, ${ }^{7}$ Grethe Myklebust, ${ }^{1}$ Tommi Vasankari, ${ }^{1}$ Pekka Kannus, ${ }^{1}$ Jari Parkkari. ${ }^{1}$ Tampere Research Center of Sports Medicine, UKK Institute, Finland; ${ }^{2}$ Faculty of Sport and Health Sciences, University of Jyväskylä, Finland; ${ }^{3}$ Sport Injury Prevention Research Centre, Faculty of Kinesiology, University of Calgary, Canada; ${ }^{4}$ Alberta Children's Hospital Research Institute, University of Calgary, Canada; ${ }^{5} \mathrm{McC}$ aig Institute for Bone and Joint Health, University of Calgary, Canada; ${ }^{6}$ Faculty of Information Technology, University of Jyäskylä, Finland; ' Oslo Sports Trauma Research Center, Department of Sports Sciences, Norwegian School of Sport Sciences, Norway
}

\subsection{6/bjsports-2019-scandinavianabs.5}

Introduction Prospective studies investigating risk factors for low back pain (LBP) in young athletes are limited. The aim of this prospective cohort study was to investigate the association between LBP and selected biomechanical factors and postural stability during dynamic movement tasks in young athletes.
Materials and methods 396 young floorball and basketball players (mean age $15.8 \pm 1.9$ ) were included and followed prospectively for 1-3 years (2011-2014). In the beginning of every study year the players were tested. The physical tests included single-leg squat (SLS), single-leg vertical drop jump (SLVDJ), vertical drop jump (VDJ) and Star Reach Excursion Balance Test (SEBT). Individual exposure time and LBP resulting in time-loss were recorded prospectively. Cox's proportional hazard models with mixed effects and time-varying risk factors were used.

Results In SLVDJ landing with non-dominant leg, the risk for general LBP and non-traumatic LBP in specific, was significantly higher with increased femoral adduction (HR 1.10, 95\% CI 1.02-1.19 and HR 1.12, 95\% CI 1.03-1.22, respectively) and significantly lower with increased in femur-pelvic angle (FPA; angle-between pelvis and femur) (HR 0.93, 95\% CI $0.88-0.99$ and HR 0.92 , 95\% CI $0.86-$ 0.99, respectively). However, the ROC analysis revealed poor combined sensitivity and specificity for femoral adduction and for FPA.

Conclusions Increased femoral adduction and decreased FPA during SLVDJ landing are associated with risk of LBP in young team ball players. However, the identified risk factors do not discriminate players with or without future LBP well enough and therefore further studies on effect of neuromuscular training on lumbo-pelvic control and LBP incidence are warranted.

\section{IMPROVED DAILY MOVEMENT PATTERNS IN AN ACCELEROMETER-ASSESSED 8-WEEKS EXERCISE PROJECT IN OLDER ADULTS}

${ }^{1}$ Manne Godhe*, ${ }^{1}$ Örjan Ekblom, 1,2 Maria Ekblom, ${ }^{1}$ Marjan Pontén, 1,2MD Eva Andersson. ${ }^{1}$ The Swedish School of Sport and Health Sciences, Sweden; ${ }^{2}$ The Department of Neuroscience, Karolinska Institutet, Sweden

\subsection{6/bjsports-2019-scandinavianabs.6}

Background Many beneficial health outcomes for elderly are associated with sedentary (SED) and physical activity behavior (PA). However, research is scarce regarding how elderly-interventions can improve various SED- and PA-behavior.

Purpose The aim with this study was to determine if eightweeks of regular supervised exercise would have any effect on movement-patterns in elderly. For comparison, a control group, not receiving supervised exercise, performed similar pre- and end-tests intervals. Reliability of elderly accelerometer measures, in two separate pre-tests was also investigated.

Methods Seventy-eight elderly exercised (65-91 years) and 43 participated in a control group (65-88 years). The exercise group had combined aerobic and strength-training, twice/w à 1 hour. Accelerometer assessments for the exercise group were performed three times; Pre- 1 and Pre- 2 and one test in the last week of the 8-week-exercise-period. The controls made one pre-test and one end-test at corresponding spring time intervals, without receiving any supervised physical activity.

Results The exercise-group significantly improved from Pre-1 to $\quad$ End-test moderate-to-vigorous-PA-intensity (MVPA $+41 \mathrm{~min} /$ week $=+0.8 \%$ of-total-daily-wear-time), lightintensity $\quad(\mathrm{LPA}+228 \mathrm{~min} / \mathrm{w}=+3.9 \%)$ total-SED-time $(-254 \mathrm{~min} / \mathrm{w}=-4.7 \%)$ and SED-bouts-à-10 $\mathrm{min}(-320 \mathrm{~min} /$ $\mathrm{w}=-5.7 \%)$. The controls showed no significant improvements 
from pre-1-to-End-tests, except for LPA $(+2.3 \%)$ and totalSED-time (and -2.7\%). Total-daily-counts in the End-test increased for controls $(+6,7 \%)$ but significantly more for the exercise group $(+17,3 \%)$. Between the exercise groups' two pre-tests, no significant difference was observed, except for total-PA $(+4.6 \%)$ and SED-total $(-2.4 \%)$.

Conclusion Elderly participating in supervised exercise increase PA- and decrease SED-time, which is of importance because it is linked with improved health for elderly, longevity and potent socioeconomic gains.

\section{\begin{tabular}{l|l}
7 & PLATELET RICH PLASMA FOR ACUTE ACHILLES TENDON
\end{tabular} RUPTURE: A DOUBLE-BLIND, MULTICENTRE, RANDOMISED, PLACEBO-CONTROLLED TRIAL}

${ }^{1}$ David Keene* ${ }^{2}$ Joseph Alsousou, ${ }^{3}$ Paul Harrison, ${ }^{1}$ Philippa Hulley, ${ }^{1}$ Susan Wagland ${ }^{1}$ Scott Parsons, ${ }^{1}$ Jacqueline Thompson, ${ }^{1}$ Heather $\mathrm{O}^{\prime}$ Connor, ${ }^{1}$ Michael Schlüssel, ${ }^{1}$ Susan Dutton, 'Sarah Lamb, 'Keith Willett. 'University of Oxford, Nuffiled Department of Orthopaedics, Rheumatology and Musculoskeletal Sciences, UK; ${ }^{2}$ University of Liverpool, Institute of Translational Medicine, UK; ${ }^{3}$ University of Liverpool, Institute of Inflammation and Ageing, UK

\subsection{6/bjsports-2019-scandinavianabs.7}

Introduction Platelet Rich Plasma (PRP), an autologous supraphysiological concentration of platelets from whole blood, has demonstrated positive cellular and physiological effects on healing in the laboratory. However, evidence from robust clinical trials is lacking. We aimed to determine the clinical efficacy of PRP for treating acute Achilles tendon rupture.

Materials and methods 230 adults starting non-surgical management within 12 days of rupture were randomised to PRP injection or dry needle insertion, under local anaesthetic. Participants were blinded and received standardised rehabilitation. Blinded outcome assessments were at 4, 7, 13, and 24 weeks. Primary outcome: muscle-tendon function assessed by work performed during the heel-rise endurance test (HRET), measured with the Limb Symmetry Index (LSI, 0\%-100\%) at 24 weeks. Secondary outcomes: Achilles Tendon Rupture Score (ATRS), quality of life, pain and goal attainment.

Results Of 230 participants, 114 were allocated to PRP injection (103 received PRP), 116 were allocated to and received placebo. At 24 weeks, 201/230 (87\%) completed the HRET and 214/230 (93\%) completed patient-reported outcomes. Participant characteristics between the groups were similar. There was no difference between groups at 24 weeks in LSI (mean difference $=-4.373 ; \quad 95 \%$ CI $-11.217,2.471 ; \quad \mathrm{p}=0.195)$. There were no differences in the secondary outcomes and adverse event rates.

Conclusion This study design and standardised PRP preparation secure robust clinical trial evidence for PRP in managing Achilles tendon rupture, and suggest that PRP offers no patient benefit. Use of PRP in soft tissue injuries must be questionable unless supported by equally robust evidence indicating positive outcomes.

\section{THE EFFECT OF CHANGE OF DIRECTION ANGLE ON KNEE MECHANICS - IMPLICATIONS FOR ACL INJURY}

Ayman Alhammad*, Lee Herrington, Paul Jones, Ritchard K Jones. School of Health Sciences, University of Salford, UK

10.1136/bjsports-2019-scandinavianabs.8
Introduction Change of direction (COD) manoeuvres are important for many field sports, however they are unfortunately associated with non-contact ACL injuries. Although players frequently $\mathrm{COD}$ at $>90^{\circ}$ angles, limited knowledge is available on knee joint kinematics and kinetics during COD at $90^{\circ}$ and $135^{\circ}$ and whether limb preference impacts knee mechanics during COD at these angles, which formed the aims of this study.

Methods Healthy male recreational soccer players were recruited to take part in the study. 3D kinematics and kinetics were collected during COD manoeuvres at $90^{\circ}$ and $135^{\circ}$. Running speed was controlled at $4 \pm 0.4 \mathrm{~m} / \mathrm{s}$ and $3.5 \pm 0.3 \mathrm{~m} / \mathrm{s}$, respectively. To determine differences on variables associated with ACL risk; knee abduction angle and moment, across cutting angles and preferred legs, a paired sample t-test was conducted using a Holm method correction, $\alpha=(0.05 / \quad(8$ comparisons - rank +1 ).

Results 36 individuals took part in the study $(24.25 \pm 6.21$ years, $1.72 \pm 0.06 \mathrm{~m}$ and $66.41 \pm 10.83 \mathrm{~kg}$ ). COD at $135^{\circ}$ showed greater knee abduction angles and moments than at $90^{\circ}$ but with similar peak VGRF. There were no differences between preferred and non-preferred legs, apart from the increased knee flexion angle during COD manoeuvres at $90^{\circ}$ in the non-preferred leg.

Conclusion In male recreational soccer players, sharper cutting angles place the knee at more risk for ACL injuries with little asymmetry between preferred and non-preferred limbs. Sharper angles of examination should be utilized in the evaluation of individuals.

\section{CHANGE IN PATIENT-REPORTED OUTCOMES FOLLOWING MENISCAL REPAIR COMPARED WITH RESECTION IN YOUNG ADULTS: SECONDARY ANALYSES FROM A PROSPECTIVE COHORT STUDY}

${ }^{1}$ Kenneth Pihl ${ }^{*}$, ${ }^{2,3}$ Martin Englund, ${ }^{4,5}$ Robin Christensen, ${ }^{6}$ Stefan Lohmander, ${ }^{7}$ Uffe Jørgensen, ${ }^{8}$ Nis Nissen, ${ }^{8}$ Jakob V Fristed, ${ }^{1}$ Jonas B Thorlund. ${ }^{1}$ Department of Sports Science and Clinical Biomechanics, University of Southern Denmark, Denmark; ${ }^{2}$ Lund University, Faculty of Medicine, Department of Clinical Sciences Lund, Orthopedics, Clinical Epidemiology Unit, Sweden; ${ }^{3}$ Clinical Epidemiology Research and Training Unit, Boston University School of Medicine, USA; ${ }^{4}$ Musculoskeletal Statistics Unit, The Parker Institute, Copenhagen University Hospital, Denmark; ${ }^{5}$ Department of Rheumatology, Odense University Hospital, Denmark; ${ }^{6}$ Lund University, Faculty of Medicine, Department of Clinical Sciences Lund, Orthopedics, Sweden; ${ }^{7}$ Department of Orthopedics and Traumatology, Odense University Hospital, Denmark; ${ }^{8}$ Department of Orthopedics, Lillebaelt Hospital, Denmark

\subsection{6/bjsports-2019-scandinavianabs.9}

Introduction In young adults, meniscal repair may be an alternative to resection. We compared change in patientreported outcomes from before to 52 weeks after arthroscopic surgery in younger patients having either meniscal repair or resection.

Materials and methods Patients aged 40 or younger from the Knee Arthroscopy Cohort Southern Denmark (KACS) study undergoing arthroscopic meniscal surgery. Patients completed the Knee injury and Osteoarthritis Outcome Score (KOOS) online at pre-surgery, 12 and 52 weeks follow-up. Between-group differences in change in a composite of 4 of 5 KOOS subscales $\left(\mathrm{KOOS}_{4}\right)$ from baseline to 52 weeks were analyzed using repeated measures mixed linear models. 